\title{
Update from RCP Quality Improvement: Quality improvement faculty and partners network
}

This part of the Future Healthcare Journal is where you will find regular updates on the work of the RCP Quality Improvement programme.

We very much welcome your feedback. If you have any comments, or would like to be involved in the work of the programme, please contact RCPQI@rcplondon.ac.uk.

The work of the Royal College of Physicians (RCP) Quality Improvement (QI) programme is collaborative in its nature, and reliant on joint working with a number of colleagues inside and outside of the college. People and organisations will connect, collaborate and contribute to RCPQI's work and aims to differing degrees. To enable this we are developing our partners network and faculty.

RCPQI aims to make quality improvement easily accessible. It supports physicians and their teams to develop and provide safe, timely, evidence-based, efficient and person-centred care, to achieve the RCPs strategic aim to improve quality. ${ }^{1}$ This work is delivered through six work streams (Fig 1), which are supported by a faculty of quality improvement experts.

\section{RCPQI partners network}

Building on the success of the Future Hospital programme partners network we are now connecting people we work with and who support our aims though RCPQI partners network. The network is an active and evolving community of people who are quality improvement champions. We want to draw on their expertise, experience and enthusiasm to inspire a social movement towards the realisation of quality improvement as a core part of professionalism for all. Those who engage with RCPQI either through attendance at events or participation in one of the programmes workstreams are invited to join the RCPQI partners network. Partner organisations such as other royal colleges, specialist societies and other organisations working on quality improvement can also join. We are sharing insights from the learnings of the programme, we are promoting innovative practice and sharing experiences of leading improvement, we are bringing the improvement community together and we are providing information and resources to enable others to lead improvement in their areas. Many members of our network are also active members in other networks, in particular the $Q$ Community. ${ }^{2}$

The RCPQI faculty was launched in November 2017, so it is still in its infancy. In the first year faculty members contributed to our development in many ways, and helped to determine the next phase of how we work together. Some were able to teach and coach teams in a 'breakthrough' collaborative, others contributed to our thinking with partners in focussed development workshops or delivered education sessions on QI (Fig 2); all valued connecting with each other formally and informally. Some haven't yet found a satisfactory way to contribute with all their other commitments.

Joanne Watson, deputy medical director at Torbay and South Devon NHS Foundation Trust, led the diabetes inpatient collaborative and commented:

Working on the collaboratives as a QI faculty member is really rewarding - connecting with teams, working on issues which are important to them and seeing results, changed attitudes and perspectives. At the end of each day, you know you have accomplished a day's work which has had impact.
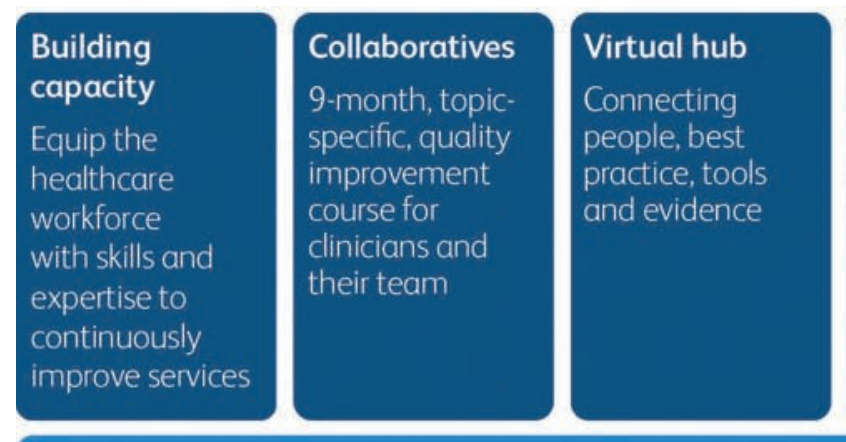

Leadership for improvement

Develop medical leaders who can influence and embed a culture of quality and continuous improvement
Research and development

Develop, adapt and design new improvement methods and knowledge
Bespoke support

Provide expert assessment and support in tackling particular organisational and service challenges 


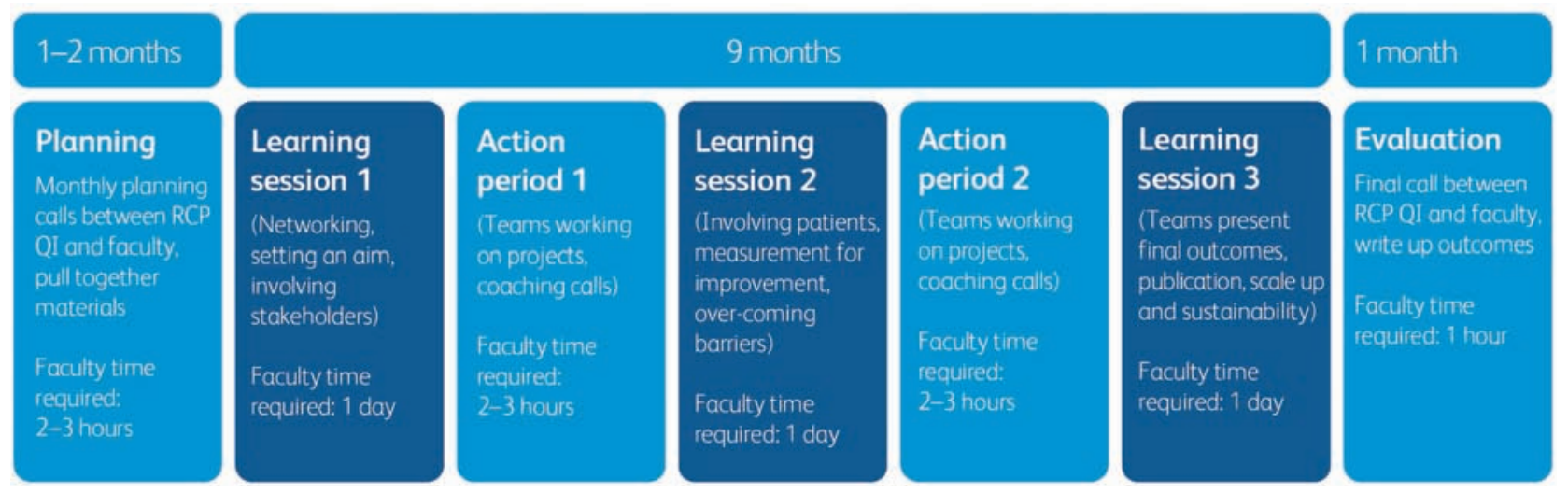

Fig 2. Structure for collaboratives to deliver training.

Saurabh Chaudhri, acute physician and nephrologist at Barts Health, contributed to collaboratives and workshops:

The opportunity to facilitate the collaborative gave me an excellent chance to engage with teams from different sites and encourage use of QI methodology in achieving their goals. I could see first-hand the energy and passion that teams working as part of a collective bring to the table despite the environment of resource limitations. It was great to have like-minded people to problem solve with and support each other in achieving their goals. It is very stimulating and encouraging.

\section{Next steps}

The faculty have also been very clear that they are at different stages of developing their QI practice skills, and need personal development support. At our second faculty development day, we agreed that a structured and managed approach to skill and practice development for QI was required for faculty as well as all physicians and teams.

We are now developing RCPQI faculty and partners network to meet this wide range of needs. Many and increasing numbers of physicians and team members want to keep connected around QI and will be part of the partners network. Some will remain faculty members and, in agreement with their employers, will commit a small number of days per year to RCPQI programmes; we expect this number to grow significantly. A smaller number who have particular experience, expertise and can contribute more time will become RCPQI faculty fellows, and will be contracted for their increased time with RCPQI.

RCP commits to supporting the development of RCPQI faculty members and fellows, and will formalise arrangements to do this.
RCPQI faculty and partners network is a growing community of practice and influence. Our vision is that quality improvement practice becomes a natural part of professional practice for physicians and team members, that all departments and trusts / health boards will have RCPQI supported clinical leads for QI, and that RCP regions will have QI leads to guide others within the region and connect together across regions.

The next steps in this for 2019 are:

$>$ diversifying and growing a multidisciplinary faculty and network

> appointing regional RCPQI faculty leads

> supporting chief registrar alumni to become future RCPQI faculty members

> delivering a development programme for RCPQI faculty members and fellows.

Monthly newsletters for those in the partners network and faculty keep people connected and informed and highlight opportunities to be involved with RCP and other QI related programmes. They also spotlight individual faculty members and their work.

If you would like to be part of RCPQI partners network, or would like to explore being a faculty member or fellow then please contact us directly via email at rcpqi@rcplondon.ac.uk.

John Dean

Clinical director for quality improvement and patient safety, Royal College of Physicians

\section{References}

1 Royal College of Physicians. Defining the RCP's approach to quality. London: RCP. www.rcplondon.ac.uk/defining-rcp-s-approach-quality [Accessed 4 December 2018].

2 The Health Foundation. About Q. London: The Health Foundation. https://q.health.org.uk/about/ [Accessed 4 December 2018]. 